\title{
Volterra Models in Load Leveling Problem
}

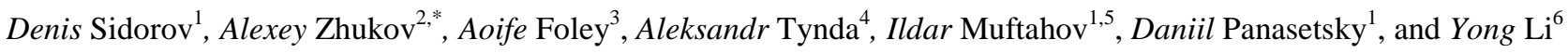 \\ ${ }^{1}$ Melentiev Energy Systems Institute, Siberian Branch of Russian Academy of Sciences, Irkutsk, Russia \\ ${ }^{2}$ Institute of Solar-Terrestrial Physics, Siberian Branch of Russian Academy of Sciences, Irkutsk, Russia \\ ${ }^{3}$ School of Mechanical and Aerospace Engineering, Queen's University Belfast, Belfast, UK \\ ${ }^{4}$ Department of Computer Engineering, Penza State University, Penza, Russia \\ ${ }^{5}$ Irkutsk Division of Main Computer Centre, JSC Russian Railways, Irkutsk, Russia \\ ${ }^{6}$ College of Electrical and Information Engineering, Hunan University, Changsha, China
}

\begin{abstract}
Further growth in renewable energy and planned electrification and decentralization of transport and heating loads in future power systems will result in a more complex unit commitment problem (UCP). This paper proposes an adaptive approach to load leveling problem using novel dynamic models based on the Volterra integral equations of the first kind with piecewise continuous kernels. This approach employs a direct numerical method. The considered collocation-type numerical method has the second-order accuracy and enjoys self-regularization properties, which is associated with confidence levels of system demand. This adaptive approach is suitable for energy storage optimization in real time. The efficiency of the proposed methodology is demonstrated on the Single Electricity Market of the Island of Ireland.
\end{abstract}

\section{Introduction}

Development of reliable methods for optimized energy storage and generation is one of the most imminent challenges in modern power systems.

The traditional least-cost dispatch of available generation based on partial differential equations to meet load will no longer be fit for the purposes for a number of reasons, including further decentralization of power systems (including transport and heating loads) and renewable energy use. A number of alternative methods have been examined to solve this challenging problem. For example, Lagrangian relaxation, mixed integer linear programming, particle swarm optimization, evolutionary methods, and genetic algorithms, [1]. These methods have many advantages and disadvantages compared to the traditional approach. However, they have not been applied by industry due to the complicated heuristics and computational constraints. The increasing complexity of the UCP can be seen in power systems with large wind penetrations. Several European countries, such as Germany, Spain, Denmark, and Ireland, have already reached a high level of installed wind power, while others such as China and the USA are showing fast rates of development. For example, wind farms in Eastern Germany during strong wind conditions can supply up to $12 \mathrm{GW}$, which is more than all of the coal- and gas-fired power plants in that part of the country combined. The Irish transmission system operator (TSO), currently restricts the instantaneous proportion of total generation allowed from non-synchronous sources, such as wind turbines, to $50 \%$ maximum, in order to maintain sufficient system inertia [2]. This can result in wind curtailment at any time, for example during high wind speeds and low electricity demand or during sudden high speeds (i.e. ramps) during periods of high electricity demand, to maintain system stability. This has an economic impact on the power system with increased operational costs through additional grid balancing charges. Thus , large-scale integration of wind power is challenging in terms of power system management. This increase in the overall cost of the produced energy limits the benefits of using renewable energy resources.

A way of reducing the uncertainty associated with wind power production is to use forecasting tools. Wind power and load forecasting, over lead times of up to 48 hours, are useful for the market operator to create dayahead unit commitment and economic dispatch schedules. Many TSOs also use shorter-term wind forecasts to draw upon system reserves for short-term balancing. Increasing the value of wind generation through the improvement of prediction systems performance is recognized as one of the priorities in wind energy research for the coming years. In fact, a reduction in the forecast errors by a fraction of a percent can lead to a substantial increase in trading profits. For example, according to [3, 4], an increase of only $1 \%$ in forecast error caused an increase of 10 million pounds in operating costs per year for one electric utility in the UK.

The unit commitment problem for optimized energy storage and generation has attracted many researchers in the last decade. It involves solving various problems including energy loss minimization, load leveling (peak load shaving/shifting), load forecasting and overall energy infrastructures management. In [5], a

Corresponding author: zhukovalex13@gmail.com 
methodology for an optimal placement of energy storage is designed for energy loss minimization through peak shaving in the presence of renewable distributed generation. The agent-based distributed control scheme for real-time peak power shaving is proposed in [6]. A price-based control system in conjunction with energy storage is analyzed in [7] for two applications: space heating in buildings and domestic freezers. It is shown that savings of up to $62.64 \%$ per day can be achieved based on New Zealand electricity rates. The review of China coal-fired power units peak regulation with a detailed presentation of the installed capacity, peak shaving operation modes and support policies is given in [8]. The authors of [9] cover the life cycle cost analysis of various energy storage technologies such as pumped hydropower storage, compressed air energy storage, flywheel, electrochemical batteries supercapacitors, and hydrogen energy storage. An analysis of time-of-use energy cost management using distributed electrochemical storages and optimum community energy storage system are correspondingly given in [10] and [11]. An optimized model of hybrid battery energy storage system based on the cooperative game model is proposed in [12]. In [13] , the effectiveness of wind turbine, energy storage, and demand response programs in the deterministic and stochastic circumstances and influence of uncertainties of the wind, price, and demand are assessed in the Energy Hub planning. It is worth noting that UCP solver is an integral part of Energy Hub which manages interconnection of heterogeneous energy infrastructures, including renewable energy resources.

This paper presents a methodology developed to solve the UCP using the systems of Volterra integral equations (VIE) of the first kind considering future power systems with battery energy storages of various efficiencies using load forecasts over lead times of 24 hours. These integral equations efficiently solve such an inverse problem given both the time-dependent efficiencies and the availability of generation/storage technologies. The state-of-the-art short-term forecasting methods can be found in [14]. In [15], the authors discuss the state-of-the-art short-term electric load forecasting methods. Power load can be forecast using many different techniques such as classical statistical methods of time series analysis, regressive models or methods based on artificial intelligence. The state-of-theart machine learning algorithms include Random Forest (RF) [16], Gradient Trees Boosting (GTB) [17] and SVM with a radial kernel (SVM RK) [18]. In this case , time series like approach was applied, i.e. it is assumed that there are relationships between the recently obtained values and future load values.

Volterra equations naturally take into account the evolutionary character of power systems. The kernels of employed equations have jump discontinuities along the continuous curves starting at the origin. Such piecewise continuous kernels $\mathrm{K}(\mathrm{t}, \mathrm{s})$ take into account both efficiencies of the different storing technologies and their proportions (which could be time-dependent) in the total power generation. Efficiencies of the different storages [9] may depe nd not only on their age and use duration (correspondingly defined by variables $t$ and $s$ ) but also on the state of charge. This can be examined using nonlinear VIE. In this paper, we demonstrate how these novel models can be applied to solve the load leveling problem.

This paper is structured into four sections. Section I presents the background on VIE. Section II develops the VIE for the UCP. Section III describes the results of the numerical experiments on real data from the Irish test system. Section IV draws a brief conclusion

\section{Volterra model}

The proposed collocation-type numerical method has the second-order accuracy and enjoys self-regularization properties as mesh step is associated with confidence levels of system demand. The proposed approach is suitable for energy storage control in real time.

Studies of linear Volterra integral equations of the first kind with piecewise continuous kernels (1) were initiated in [20, 21] and pursued in [19], [22-25] followed by monograph [15]. In general, VIE of the first kind can be solved by reduction of the equations of the second kind. The regularization algorithms developed for Fredholm equations can also be applied, as well as direct discretization methods. On the other hand, it is known that solutions to integral equations of the first kind can be unstable and this is a well known ill-posed problem. This is due to the fact that the Volterra operator maps the considered solution space into its narrow part only. Therefore, the inverse operator is not bounded and it is necessary to assess the proximity of the solutions and the proximity of the right-hand side using different metrics: the proximity of the right-hand side should be in a stronger metric. Moreover, as shown in [15], solutions to the VIE can contain arbitrary constants and can be unbounded as $t \rightarrow 0$. The problems of existence, uniqueness and asymptotic behavior of VIE solutions are explored in [20, 21, 24].

The theory of integral models of evolving systems was initiated by L. Kantorovich [26], R. Solow [27] and V. Glushkov [28] in the mid-20th century. It is well known that Solow publication [27] on vintage capital model led him to the Nobel prize in 1987 for his analysis of economic growth.

Such a theory leads to the VIEs of the first kind where bounds of the integration interval can be functions of time. These models take into account the memory of a dynamical system when its past impacts its future evolution. The memory is implemented in the existing technological and financial structure of physical capital (equipment). The memory duration is determined by the age of the oldest capital unit (e.g. storages) still employed. There are several approaches to numerically solving VIE of the first kind. One of them is to apply classical regularizing algorithms developed for Fredholm integral equations of the first kind [29]. However, the problem reduces to solving algebraic systems of equations with a full matrix, an important advantage of the VIE is lost and there is a significant increase in arithmetic complexity of the algorithms. The second 
approach is based on a direct discretization of the initial equations. Here one may face an instability of the approximate solution because of errors in the initial data (load forecast). The regularization properties of the direct discretization methods are optimal in this sense, where the discretization step is the regularization parameter associated with the error of the source data (load forecast error upper bound). The detailed description of regularizing direct numerical algorithms is given in [29]. It is worthwhile to note that it is very difficult to apply these algorithms to solve equation (1) in the form of (3) because of the kernel discontinuities (2). The adaptive mesh should depend on the curves of the jump discontinuity for each number $\mathrm{N}$ of divisions of the considered interval and, therefore, this mesh cannot be linked to the errors in the source data. It is necessary to correctly approximate the integrals.

It is worth noting that the load leveling problem in UCP has no such luxury as discretization step control because source data are measured using the fixed step only. Therefore, Lavrentiev regularization [29, 30] using parameter $\alpha$ (Table 1 ) is employed.

The object of interest is the following integral equation of the first kind

$$
\int_{0}^{t} K(t, s) x(s) d s=f(t), t \in[0, T]
$$

where the kernel $K(t, s)$ is discontinuous along continuous curves $\alpha_{i}(t), i=1,2, \ldots, n-1$, and is of the form

$$
K(t, s)= \begin{cases}K_{1}(t, s), & \alpha_{0}(t)<s<\alpha_{1}(t) ; \\ K_{2}(t, s), & \alpha_{1}(t)<s<\alpha_{2}(t) ; \\ \cdots & \\ K_{n}(t, s), & \alpha_{n-1}(t)<s<\alpha_{n}(t) .\end{cases}
$$

Here $\quad \alpha_{0}(t) \equiv 0, \alpha_{0}(t)<\alpha_{1}(t)<\cdots<\alpha_{n}(t) \equiv t$, $f(0)=0$. It is assumed that the kernels $K_{i}(t, s)$ and the right-hand side $f(t)$ in the equation (1) are continuous and sufficiently smooth functions. The functions $\alpha_{i}(t) \in \mathcal{C}^{1}[0, T]$ are not decrescent. Moreover $\alpha^{\prime}{ }_{1}(0) \leq$ $\alpha_{2}^{\prime}(0) \leq \cdots \leq \alpha_{n-1}^{\prime}(0)<1$. Rewrite equation (1) as follows

$$
\begin{gathered}
\int_{0}^{\alpha_{1}(t)} K_{1}(t, s) x(s) d s+\int_{\alpha_{1}(t)}^{\alpha_{2}(t)} K_{2}(t, s) x(s) d s+\cdots \\
\cdots+\int_{\alpha_{n-1}(t)}^{t} K_{n}(t, s) x(s) d s=f(t) \\
t \in[0, T] .
\end{gathered}
$$

The mesh nodes are introduced (not necessarily uniform) to construct a numeric solution to equation (3) on the interval $[0, T]$ (if the unique continuous solution exists) $0=t_{0}<t_{1}<t_{2}<\cdots<t_{N}=T, h=\max _{i=1, N}\left(t_{i}-\right.$ $\left.t_{i-1}\right)=\mathcal{O}\left(N^{-1}\right)$. The approximate solution is determined as the following piecewise linear function

$$
\begin{gathered}
x_{N}(t)=\sum_{i=1}^{N}\left(x_{i-1}+\frac{x_{i}-x_{i-1}}{t_{i}-t_{i-1}}\left(t-t_{i-1}\right)\right) \delta_{i}(t), \\
t \in(0, T], \\
\text { where } \delta_{i}(t)= \begin{cases}1, & \text { for } t \in \Delta_{i}=\left(t_{i-1}, t_{i}\right] . \\
0, & \text { for } t \notin \Delta_{i} .\end{cases}
\end{gathered}
$$

The coefficients $x_{i}, i=\overline{1, N}$ of the approximate solution for $x_{0}=x(0)$

$$
x_{0}=\frac{f^{\prime}(0)}{\sum_{i=1}^{n} K_{i}(0,0)\left[\alpha^{\prime}{ }_{i}(0)-\alpha{ }^{\prime}{ }_{i-1}(0)\right]}
$$

and for $x_{1}=x\left(t_{1}\right)$

$$
\begin{aligned}
& x_{1}=\frac{f_{1}}{\sum_{i=1}^{n}\left(\alpha_{i}\left(t_{1}\right)-\alpha_{i-1}\left(t_{1}\right)\right) K_{i}\left(t_{1}, \frac{\alpha_{i}\left(t_{1}\right)+\alpha_{i-1}\left(t_{1}\right)}{2}\right)} \\
& \text { and for } x_{k}=x\left(t_{k}\right), k=1, \ldots, N \\
& \qquad x_{k}=x_{k-1}+\left[\mathrm{f}_{\mathrm{k}}-\mathrm{x}_{\mathrm{k}-1} \int_{\mathrm{t}_{\mathrm{k}-1}}^{\mathrm{t}_{\mathrm{k}}} \mathrm{K}\left(\mathrm{t}_{\mathrm{k}}, \mathrm{s}\right) \mathrm{ds}-\right. \\
& \quad-\sum_{\mathrm{j}=1}^{\mathrm{k}-1}\left(\mathrm{x}_{\mathrm{j}-1} \int_{\mathrm{t}_{\mathrm{j}-1}}^{\mathrm{t}_{\mathrm{j}}} \mathrm{K}\left(\mathrm{t}_{\mathrm{k}}, \mathrm{s}\right) \mathrm{ds}+\right. \\
& \left.\left.+\mathrm{x}_{\mathrm{j}}-\mathrm{x}_{\mathrm{j}-1} \mathrm{t}_{\mathrm{j}}-\mathrm{t}_{\mathrm{j}-1} \int_{\mathrm{t}_{\mathrm{j}-1}}^{\mathrm{t}_{\mathrm{j}}}\left(\mathrm{s}-\mathrm{t}_{\mathrm{j}-1}\right) \mathrm{K}\left(\mathrm{t}_{\mathrm{k}}, \mathrm{s}\right) \mathrm{ds}\right)\right] / \\
& \left.\qquad \frac{1}{t_{k}-t_{k-1}} \int_{t_{k-1}}^{t_{k}}\left(s-t_{k-1}\right) K\left(t_{k}, s\right) d s\right\} .
\end{aligned}
$$

The integrals in (7) can be approximated using the midpoint quadratures based on auxiliary mesh nodes so that the values of the functions $\alpha_{i}\left(t_{j}\right)$ are a subset of the set of this mesh points at each particular value of $N$. The error of this approximation method is $\varepsilon_{N}=\| x(t)-$ $x_{N}(t) \|_{C_{[0, T]}}=\mathcal{O}\left(1 / N^{2}\right)$.

\section{Results of numerical experiments on real data}

In order to evaluate the proposed load leveling method, the power load time series are taken from the Single Electricity Market of the Island of Ireland (SEM) [31]. The time interval is one week from 25.04.2016 till 01.05.2016. It is assumed that part of the batteries is eventually removed from service causing efficiency reduction up to $15 \%$. This phenomen on is described below by the coefficients of the Volterra kernel.

\subsection{Load forecasting}

The RF, GTB and SVM RK are evaluated to obtain a high accuracy model for power load forecast, which is an integral part of our approach. In order to make 24-hourahead forecast 6 load values and a day of the week were used as the most informative features. Feature importance was estimated based on RF Gini index [16]. The models' parameters were obtained using repeat ed cross-validation. RFs were used with $m$ try $=5$ and 150 trees. In the case of GTB 200 trees were employed with interaction. depth $=10, \quad$ shrinkage $=0.1 \quad$ and minobsinnode $=10$. As for SVM, the parameters were selected as sigma $=0.4428387$ and $C=128$. The performance of the methods was assessed using the mean absolute error (MAE) and root mean square error (RMSE).

Fig. 1 shows an actual load and its forecasts with a 24-hour lead interval for SEM. Here the solid green curve shows base generation (without storage), the solid blue curve stands for actual load, the green dotted line corresponds to an RF-based forecast, the SVM forecast is marked with a dashed red line, black dashed dots stand for GTB. All exploited methods show similar errors. 


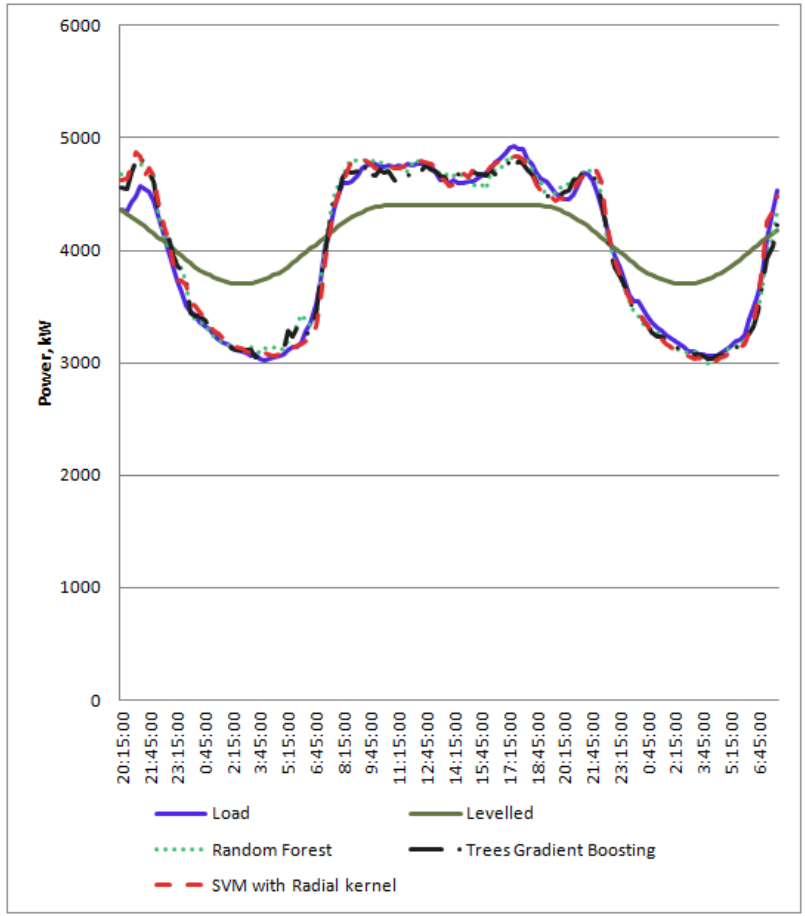

Fig. 1. Load forecasts with a 24 -hour lead interval, actual load (blue curve) and available base generation (dark green line) for SEM

\subsection{Load leveling}

For the sake of simplicity, the single virtual grid energy storage unit is considered. This unit consists of three components of various efficiencies. We model this process using VIE (1) with piecewise continuous kernel

$K(t, s)=\left\{\begin{array}{ll}1, & 0<s<t / 4, \\ 0.9, & t / 4<s<3 t / 4, \\ 0.85, & 3 t / 4<s<t,\end{array} \quad t \in[0, T]\right.$.

Here coefficients $1,0.9$ and 0.85 correspond to $100 \%, 90 \%$ and $85 \%$ efficiencies for the storage components, see e.g. [9]. Integration limits define time share of each component contributing to the charge/discharge strategy.

We ignore distribution system losses and do not take into account the storage unit location and grid topology.

Table 1. Charge/discharge errors for various $\alpha$ using SVM forecast for SEM

\begin{tabular}{c|ccc}
\hline & SVM & $\begin{array}{c}\text { SVM with } \\
\alpha=0.4217\end{array}$ & $\begin{array}{c}\text { SVM with } \\
\alpha=0.9998\end{array}$ \\
\hline RMSE & 194.6 & 142.48 & 145.52 \\
\hline MAE & 129.09 & 95.64 & 107.62 \\
\hline
\end{tabular}

Fig. 2 shows charging/discharging strategies using various 24-hour forecasts and actual data for SEM. Here the bars mark the strategy based on actual load, the green dotted curve shows the strategy using an RF forecast, the SVM based forecast strategy is marked with a dashed red line, black dashed dots stand for GTB. The curves show the strategies defined using Lavrentiev regularization parameter $\quad \alpha=0.4217 \quad$ (obtained manually).

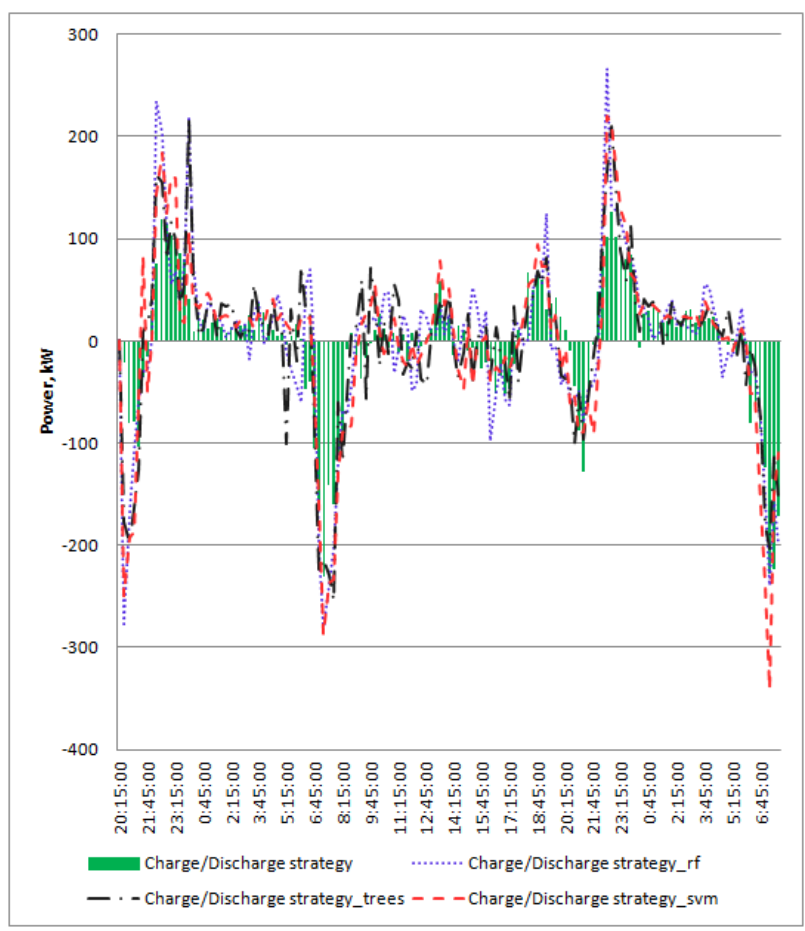

Fig.2. Charge/discharge strategies using various 24- hour forecasts compared with actual data based benchmark strategy (bars) for SEM

\section{Conclusions}

This paper presents the first multi-vector energy analysis for the interconnected energy systems of Great Britain and Ireland.

A novel mathematically grounded approach to the load leveling problem is proposed using Volterra models and tested on the Single Electricity Market of the Island of Ireland. Such evolutionary models take into account both the time-dependent efficiency and the availability of generation/storage of each energy storage technology in the power system. The SEM is a power system with high wind power penetration and much unpredictability due to the inherent variability of wind. The problem of efficient charge/discharge strategies is reduced to sol ving integral equations and their systems. The numerical methods are proposed for such equations to find the available storage dispatch schedules. The proposed method is applied to real data demonstrating its effectiveness. More accurate prediction can be achieved by including more representative features and handling concept drift as suggested in [32]. Our current research addresses load leveling given the location of storages and state of the load influence on the storage efficiency modeled using the nonlinear VIE.

\section{Acknowledgment}

This research was supported by RFBR Grant 18-3100206. 


\section{References}

1. N. I. Voropai, A. Z. Gamm, A. M. Glazunova, P. V. Etingov, I. N. Kolosok, E. S. Korkina, V. G. Kurbatsky, D. N. Sidorov, V. A. Spiryaev, N. V. Tomin, R. A. Zaika, and B. Bat-Undraal, Application of Meta-Heuristic Optimization Algorithms in Electric Power Systems, pp. 564-615. IGI Global, 2013.

2. "System Operator of Northern Ireland." http://www.soni.ltd.uk/Operations/sg/DS3/. Accessed: 2016-01-07.

3. .D. Bunn and E. Farmer, "Comparative models for electrical load forecasting," Int. J. Forecast, vol. 2, pp. 501-505, 1985.

4. L. Soares and M. Medeiros, "Modeling and forecasting short-term electricity load: a comparison of methods with an application to Brazilian data," Int. J. Forecast, vol. 24, pp. 630-644, 2008.

5. V. Kalkhambkar, R. Kumar, and R. Bhakar, "Energy loss minimization through peak shaving using energy storage," Perspectives in Science, pp. -, 2016. In press.

6. D. D. Sharma, S. Singh, and J. Lin, "Multi-agent based distributed control of distributed energy storages using load data," Journal of Energy Storage, vol. 5, pp. 134 - 145, 2016.

7. R. Barzin, J. J. Chen, B. R. Young, and M. M. Farid, "Peak load shifting with energy storage and pricebased control system," Energy, vol. 92, Part 3, pp. 505 - 514, 2015. Sustainable Development of Energy, Water and Environment Systems.

8. Y. Gu, J. Xu, D. Chen, Z. Wang, and Q. Li, "Overall review of peak shaving for coal-fired power units in China," Renewable and Sustainable Energy Reviews, vol. 54, pp. 723 - 731, 2016.

9. B. Zakeri and S. Syri, "Electrical energy storage systems: A comparative life cycle cost analysis," Renewable and Sustainable Energy Reviews, vol. 42, pp. 569 - 596, 2015.

10. G. Graditi, M. Ippolito, E. Telaretti, and G. Zizzo, "Technical and economic assessment of distributed electrochemical storages for load shifting applications: An Italian case study," Renewable and Sustainable Energy Reviews, vol. 57, pp. 515 - 523, 2016.

11. D. Parra, S. A. Norman, G. S. Walker, and M. Gillott, "Optimum community energy storage system for demand load shifting," Applied Energy, vol. 174, pp. 130 - 143, 2016.

12. X. Han, T. Ji, Z. Zhao, and H. Zhang, "Economic evaluation of batteries planning in energy storage power stations for load shifting," Renewable Energy, vol. 78, pp. 643 - 647, 2015.

13. S. Pazouki and M.-R. Haghifam, "Optimal planning and scheduling of energy hub in presence of wind, storage and demand response under uncertainty," International Journal of Electrical Power \& Energy Systems, vol. 80, pp. 219 - 239, 2016.
14. N. Tomin, A. Zhukov, D. Sidorov, V. Kurbatsky, D. Panasetsky, and V. Spiryaev, "Random forest-based model for preventing large-scale emergencies in power systems," International Journal of Artificial Intelligence, vol. 13, pp. 211-228, 2015.

15. D. Sidorov, Integral dynamical models: singularities, signals, and control, vol. 87 of World Scientific Series on Nonlinear Science Series A. Singapore: World Scientific, 2015.

16. L. Breiman, "Random forests," Machine learning, vol. 45, no. 1, pp. 5-32, 2001.

17. J. H. Friedman, "Greedy function approximation: a gradient boosting machine," Annals of statistics, pp. 1189-1232, 2001.

18. A. Smola and V. Vapnik, "Support vector regression machines," Advances in neural information processing systems, vol. 9, pp. 155-161, 1997.

19. I. Muftahov, A. Tynda, and D. Sidorov, "Numeric Solution of Volterra Integral Equations of the First Kind with Discontinuous Kernels," Journal of Computational and Applied Mathematics (Elsevier), vol. 313, pp. 119-128, 2017.

20. D. Sidorov, "Volterra Equations of the First Kind with Discontinuous Kernels in the Theory of Evolving Systems Control," Studia Informatica Universalis. Paris: Hermann Publ., vol. 9, no. 3, pp. 135-146, 2011.

21. D. N. Sidorov, "On parametric families of solutions of Volterra integral equations of the first kind with a piecewise smooth kernel," Differential Equations, vol. 49, no. 2, pp. 210-216, 2013.

22. D. N. Sidorov, "Solution to Systems of Volterra Integral Equations of the First Kind with Piecewise Continuous Kernels," Russian Mathematics (Transl. from Izvestia VUZov), vol. 57, no. 1, pp. 62-72, 2013.

23. E. V. Markova and D. N. Sidorov, "Volterra Integral Equation of the First Kind with Discontinuous Kernels in the Theory of Evolving Dynamical Systems Modeling," Izvestia Irkutskogo gos. univ. Matematika, no. 2, pp. 31-45, 2012.

24. N. A. Sidorov and D. N. Sidorov, "On the solvability of a class of Volterra operator equations of the first kind with piecewise continuous kernels," Mathematical Notes, vol. 96, no. 5, pp. 811-826, 2014.

25. E. V. Markova and D. N. Sidorov, "On one integral Volterra model of developing dynamical systems," Automation and Remote Control, vol. 75, no. 3, pp. 413-421, 2014.

26. L. V. Kantorovich and V. I. Zhiyanov, "Singlecommodity dynamic model of the economy allowing for changes in asset structure in the presence of technical progress," Dokl. Akad. Nauk USSR, vol. 211, no. 6, pp. 1280-1283, 1973.

27. R. M. Solow, Mathematical Methods in the Social Sciences, ch. Investment and Technical Progress, 
pp. 89-104. Stanford, California: Stanford University Press, 1960.

28. [V. M. Glushkov, V. V. Ivanov, and V. M. Janenko, Developing Systems Modeling. Moscow: Nauka, 1983.

29. P. K. Kythy and P. Puri, Computational Methods for Linear Integral Equations. Boston: Birkhauser, 2002.

30. I. R. Muftahov, D. N. Sidorov, and N. A. Sidorov, "Lavrentiev regularization of integral equations of the first kind in the space of continuous functions," Izvestia Irkutskogo gos. univ. Matematika, no. 15, pp. 62-77, 2016.

31. "Eirgrid Group. System Information of Ireland's Power System." http://www.eirgridgroup.com. Accessed: 2016-02-05.

32. A. Zhukov, D. Sidorov, and A. Foley, "Random forest-based approach for concept drift handling," arXiv: Artificial Intelligence (cs.AI), vol. 1602.04435 [cs.AI], pp. 1-8, 2016.

33. Yang Wei, Wu YuLin, and Liu ShuHong, "An optimization method on runner blades in bulb turbine based on CFD analysis," Science China Technological Sciences, vol. 54, no. 2, pp. 338344.

34. M. Kezunovic, S. Meliopoulos, V. Venkatasubramanian, V. Vittal. Application of Time-Synchronized Measurements in Power System Transmission Networks (Springer, NY, 2014).

35. A. Mokeev, Digital Substation. URL: http://digitalsubstation.com.

36. P. Ilyshin, Energoexpert, 1, 58-62 (2015).

37. A. Mokeev, Proceedings IEEE International Conference SIBCON (2017).

38. A. Mokeev, V. Bovykin, A. Miklashevich, D. Ulyanov. Proceedings International Conference Actual trends in development of Power System Relay Protection and Automation (2015).

39. A. Gamm, A. Glazunova, Yu. Grishin, I. Kolosok, E. Korkina, Electrical Technology Russia, 6, 2-9 (2009).

40. P. Ilyshin, P. Chusovitin, Relay Protection and Automation, 4, 16-22 (2014).

41. V. Narovlyanskii, V. Kurmak, Power Plant, 3, 48-51 (2012).

42. V. Narovlyanskii, Modern Methods and Means of Prevention of Asynchronous Operation of the Power Grid (Energoatomizdat, Moscow, 2004). 